\title{
Impact of Voltage Reduction on Voltage Stability in Emergency Conditions
}

\author{
Seon-Ju Ahn, Dong-Hyun Yoo and Joon-Ho Choi \\ Chonnam National University \\ sjahn@jnu.ac.kr;136055@ejnu.onmicrosoft.com; joono@chonnam.ac.kr
}

\begin{abstract}
Voltage reduction is one of the most common measures implemented to increase power reserves in emergency conditions. Since the power consumption of a composite load depends on the voltage, the power demand can be reduced by reducing the distribution voltage. Although the voltage reduction has a positive effect on power system in terms of securing reserve power, it may have a negative impact on the voltage stability in some conditions. Thus, it is necessary to analyze the effect of voltage reduction on the voltage stability. This paper analyzes the effect of voltage reduction on the voltage stability by performing $P V$ analysis in various system conditions, such as base case on light load, heavy load, and contingency cases. In addition, we analyzed the effect of the voltage sensitivity characteristic of loads on the voltage stability.
\end{abstract}

Keywords: Voltage reduction, voltage stability, PV analysis, emergency conditions

\section{Introduction}

Power utilities have their own operation procedures on for responding to emergency conditions [1-5]. In the event of capacity shortage, power utilities implement various control actions, such as purchasing emergency energy, voltage reduction, and shedding loads [6-11]. Voltage reduction can lower the power demand quickly, and is implemented by the power utilities to manage the reliability of the system when power demand exceeds the available supply [5]. KPX (Korea Power Exchange), the Korean power system operator, also has a five-step procedure to maintain the system reliability in the event of energy shortage. In levels 2 and 3 of the procedure, KPX reduce the distribution voltage by $2.5 \%$ and $5 \%$, respectively, by controlling the transformer tap position [12].

Even though voltage reduction can increase the power reserve in emergencies, critical devices can be damaged by low voltage. Moreover, the voltage reduction may result in voltage instability in some severe system conditions. Therefore, the effects on the voltage stability should be carefully analyzed before implementing the voltage reduction.

PV analysis is the most common method for steady-state voltage stability assessment, and it was used in this study. An IEEE 25 bus benchmark system was used as a test system, and the PV analysis was performed with various system conditions, such as a light load base case, heavy load base case, and contingency cases. Moreover, to analyze the effect of voltage sensitivity characteristics of loads on the voltage stability, we repeated the PV analysis for various load ZIP parameters.

The remainder of the paper is organized as follows. In Section 2, the PV curve is explained briefly. In Section 3, the procedure for data preparation and the PV analysis 
process using PSS/E is described. Section 4 shows the PV analysis results in the light load case, and Section 5 shows the results for heavy load and contingency conditions. Finally, a conclusion is presented in Section 6.

\section{PV Analysis}

Voltage stability is the ability of a power system to maintain acceptable voltages at all buses under normal conditions and after disturbances [13-14]. One of the most common methods to analyze the voltage stability is PV analysis. The PV curve can be illustrated by considering the two-terminal network of Figure 1(a). It consists of a constant voltage source $\left(E_{s}\right)$ supplying a load $\left(Z_{L D}\right)$ through a series impedance $\left(Z_{L N}\right)$. When phasor of $E_{s}$ and line parameters are constant, the load demand is increased by decreasing $Z_{L D}$. The power transfer $\left(P_{R}\right)$ increases rapidly at first and then slowly before reaching a maximum $\left(Z_{L D} / Z_{L N}=1\right)$, after which it rapidly decreases. Eventually, if the load is increased beyond the maximum transfer power, this power system will become unstable, which causes voltage collapse.

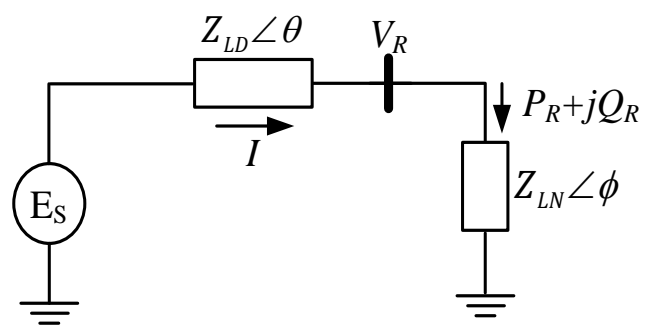

(a)

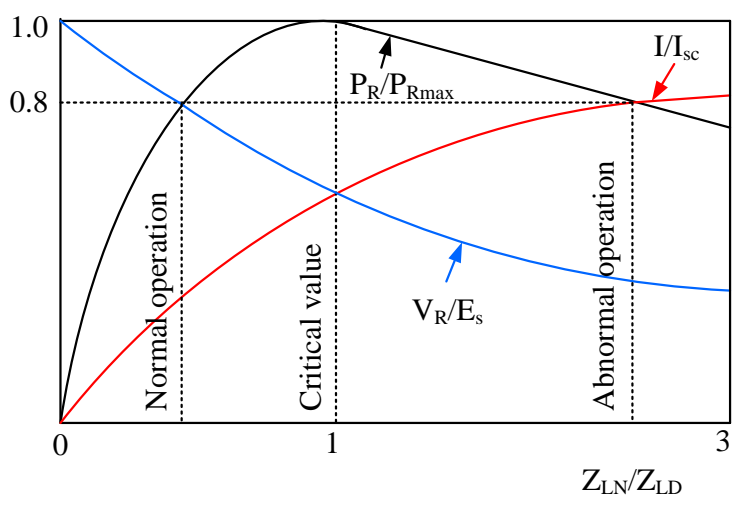

(b)

Figure 1. (a) Two-bus system and (b) Receiving end voltage, current, and power as a function of load demand

Equations (1-4) show the relationship among the $V_{R}, I, P_{R}$ in Figure 1(a) [14-16]. Figure 1(b) shows the relationship between transfer power and per unit voltage at the receiving end [14, 16-18]. 


$$
\begin{aligned}
& I=\frac{1}{\sqrt{F}} \frac{E_{s}}{Z_{L N}} \\
& V_{R}=\frac{1}{\sqrt{F}} \frac{Z_{L D}}{Z_{L N}} E_{s} \\
& P_{R}=\frac{Z_{L D}}{F}\left(\frac{E_{s}}{Z_{L N}}\right)^{2} \cos \phi
\end{aligned}
$$

where,

$$
F=1+\left(\frac{Z_{L D}}{Z_{L N}}\right)^{2}+2\left(\frac{Z_{L D}}{Z_{L N}}\right) \cos (\theta-\phi)
$$

Generally, voltage stability is usually assessed by the distance (in per unit transfer power) between the operating point on the PV curve and the limit of the same curve. Thus, in terms of voltage stability, a system is said to be more stable as the transfer power can be increased further [14, 19-22].

\section{PV Analysis Using PSS/E}

\subsection{Modified data in benchmark system}

The IEEE 25 bus system provided in the PSS/E is used as a test system. This system consists of transmission lines operating at $230 \mathrm{kV}$ or $138 \mathrm{kV}$. In the original PSS/E data, load models are assumed to be directly connected to the transmission system. However, in an actual system, loads are connected to the system through the distribution line. To analyze the effect of distribution voltage reduction, we need to modify the parameters of the benchmark system data. Firstly, we need to add the distribution voltage buses where the loads are actually connected. We used the "Split Bus" function in PSS/E, which separates the existing transmission bus into the transmission bus and $23 \mathrm{kV}$ distribution bus, and then loads are connected to the modified distribution system. After that, 17 two-winding transformers are connected between 17 new distribution buses and existing transmission buses.

\subsection{PV analysis procedures}

All load of the given system data have constant power $(P, Q)$ characteristics in the base case of the benchmark system in PSS/E, and the tap ratio of transformer has been set to 1.0. The PV analysis procedure to analyze the effect of voltage reduction according to the voltage sensitivity characteristic of the loads is as follows [23].

- Step 1: Set the load voltage sensitivity characteristic by changing the ZIP parameter of the data.

- Step 2: Change the tap ratio of all load-connected transformers to 0.9.

- Step 3: Perform the power flow using the modified system data.

- Step 4: Perform the PV analysis.

- Step 5: Repeat the steps 2 to 4, for different tap ratio settings; 0.95, 1.0, 1.05, and 1.1. 


\section{PV Analysis in Base Case of Light Load}

\subsection{Effect of transformer tap ratio}

Figure 2 shows the results of the PV analysis in the base case of light load according to changes of the tap ratio. In this test, the ZIP parameter of the loads was set as ZIP = 0.4:0.4:0.2. In Figure 2, five graphs show the PV analysis results for different tap ratios, where the $\mathrm{x}$-axis represents the incremental transfer power in $\mathrm{MW}$, and the $\mathrm{y}$ axis represents the per unit voltage at the receiving end. The results show that the maximum transfer power is increased as the tap ratio decreases. In other words, the voltage stability margin increases as the distribution voltage is reduced.

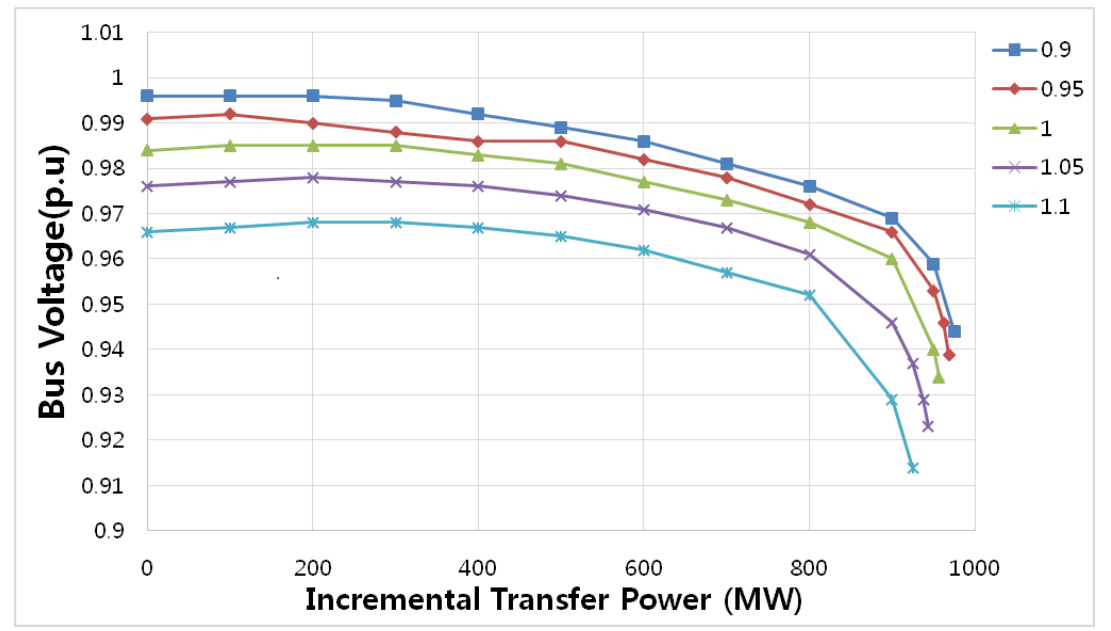

Figure 2. Results of the PV analysis according to tap ratio

\subsection{Effect of load characteristics}

When all of the load bus voltages are changed by $\Delta V$, the load variation $(\Delta P)$ can be calculated approximately using equation (5) [24].

$$
\Delta P \cong P_{0} \times(2 Z+I) \Delta V
$$

The load variation according to voltage variation is nearly proportional to the value of $2 Z+I$ according to equation (5). Therefore, the value $2 Z+I$ represents the voltage sensitivity of the load. Thus, in order to analyze the effect of the voltage sensitivity characteristic of the load on the voltage stability, we conducted a PV analysis for the following three cases. In the first two cases, the values of $2 Z+I$ are 0.9 , while the last case has a value of the 1.3.

Table 1 summarizes the results. For all three cases, both the maximum incremental transfer power $\left(P_{c p}\right)$ and the voltage at critical point $\left(V_{c p}\right)$ decrease as the tap ratio increases from 0.9 to 1.1 . This means that the voltage stability margin increases as the distribution voltage is reduced, regardless of the voltage sensitivity characteristics of the loads. The results of cases 1 and 2 are almost the same, with a small difference in $V_{c p}$, while case 3 shows quite different results. It can be concluded that if the voltage sensitivity characteristics are the same, the effects of voltage reduction on voltage stability are also the same. 
Table 1. The results of PV analysis according to load characteristics

\begin{tabular}{|c|c|c|c|c|c|c|}
\hline & \multicolumn{2}{|c|}{ Case 1: } & \multicolumn{2}{c|}{ Case 2: } & \multicolumn{2}{c|}{ Case 3: } \\
& \multicolumn{2}{|c|}{ ZIP=0.3:0.3:0.4 } & \multicolumn{2}{c|}{ ZIP=0.4:0.1:0.5 } & \multicolumn{2}{c|}{ ZIP=0.4:0.5:0.1 } \\
\hline Tap & $P_{c p}(\mathrm{MW})$ & $V_{c p}(\mathrm{pu})$ & $P_{c p}(\mathrm{MW})$ & $V_{c p}(\mathrm{pu})$ & $P_{c p}(\mathrm{MW})$ & $V_{c p}(\mathrm{pu})$ \\
\hline 0.90 & 806.250 & 0.914 & 806.250 & 0.912 & 821.875 & 0.912 \\
\hline 0.95 & 793.750 & 0.904 & 793.750 & 0.903 & 809.375 & 0.911 \\
\hline 1.00 & 775.000 & 0.900 & 775.000 & 0.900 & 790.625 & 0.890 \\
\hline 1.05 & 756.250 & 0.889 & 756.250 & 0.890 & 768.750 & 0.879 \\
\hline 1.10 & 737.500 & 0.873 & 737.500 & 0.874 & 742.359 & 0.864 \\
\hline
\end{tabular}

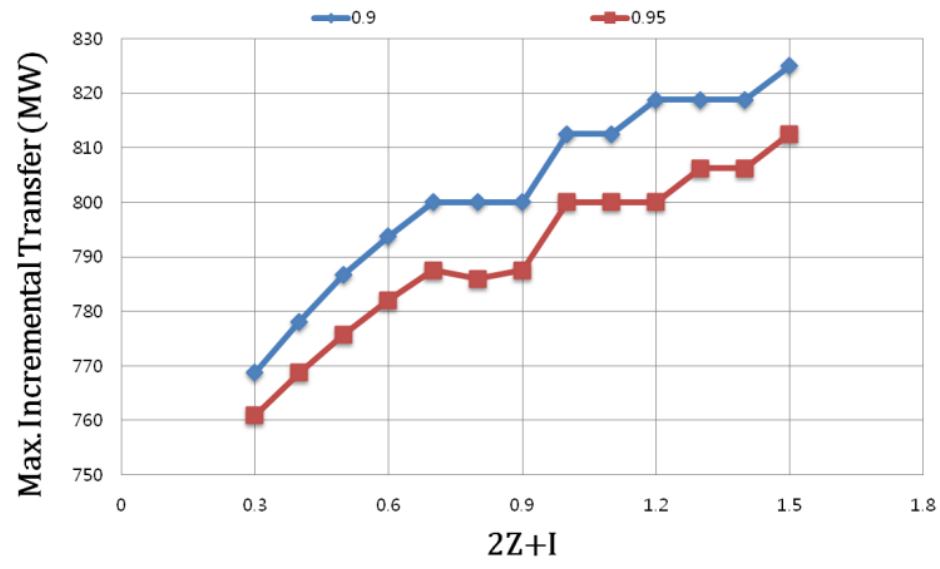

Figure 3. Relationship between voltage sensitivity characteristics and maximum incremental transfer power

In addition, in order to analyze the voltage stability according to a variety of voltage sensitivity characteristics, we conducted PV analysis for 13 different. The $2 Z+I$ values in the 13 cases are increasing by 0.1 . We repeated the same simulation for the tap ratios of 0.9 and 0.95 . Figure 3 illustrates the results. Firstly, for all $2 Z+I$ values, the maximum incremental transfer power for a tap ratio of 0.9 is always larger than that for a tap ratio of 0.95 . Secondly, for both tap ratios, the maximum incremental transfer power is increased as the voltage sensitivity value $(2 Z+I)$ increases.

\section{PV Analysis in Heavy Load and Critical Contingency Case}

Usually, voltage reduction is conducted when the power reserve is insufficient due to heavy loads or when a critical fault occurs in a system. Therefore, to analyze the effect of voltage reduction on the stability in such cases, we performed the PV analysis in heavy load conditions and some critical contingency cases. In the heavy load conditions, the power demand is increased to $4,410 \mathrm{MW}$, which is a $25 \%$ increase from the base case with light load, and the power reserve is $4.54 \%$ in this condition.

To analyze the effect of load characteristics, we considered the following three cases.

- Case $1: \mathrm{ZIP}=0.1: 0.1: 0.8$

- Case 2: ZIP = $0.4: 0.4: 0.2$,

- Case 3: ZIP = -0.1:-0.1:1.2. 
Table 2. PV analysis results of case 1

\begin{tabular}{|c|c|c|c|c|c|}
\hline \multicolumn{7}{|c|}{ Case 1: ZIP =0.1:0.1: 0.8 } \\
\hline CASE & \multicolumn{7}{|c|}{ MAX.INCR.TRANS } \\
\hline TAP & 0.9 & 0.95 & 1.0 & 1.05 & 1.1 \\
\hline BASE CASE & 768.75 & 760.94 & 752.34 & 742.97 & 732.81 \\
\hline CON 01 & 619.53 & 0 & 0 & 0 & 0 \\
\hline CON 02 & 662.72 & 655.47 & 649.22 & 642.19 & 633.59 \\
\hline CON 03 & 739.84 & 726.56 & 712.50 & 684.38 & 656.25 \\
\hline CON 04 & 801.56 & 791.41 & 781.25 & 769.53 & 757.03 \\
\hline CON 05 & 632.81 & 626.56 & 618.75 & 610.94 & 602.34 \\
\hline CON 06 & 760.94 & 752.34 & 743.75 & 733.59 & 722.66 \\
\hline CON 07 & 564.84 & 0 & 0 & 0 & 0 \\
\hline
\end{tabular}

Table 3. PV analysis results of case 2

\begin{tabular}{|c|c|c|c|c|c|}
\hline \multicolumn{7}{|c|}{ Case 2: ZIP = 0.4 : 0.4: 0.2 } \\
\hline CASE & \multicolumn{7}{|c|}{ MAX.INCR.TRANS } & 1.1 \\
\hline TAP & 0.9 & 0.95 & 1.0 & 7.05 & 739.06 \\
\hline BASE CASE & 818.75 & 805.47 & 788.28 & 767.19 & 679.69 \\
\hline CON 01 & 751.56 & 739.06 & 723.44 & 703.13 & 633.59 \\
\hline CON 02 & 691.41 & 682.81 & 670.31 & 653.91 & 735.16 \\
\hline CON 03 & 809.38 & 796.06 & 778.91 & 758.59 & 756.25 \\
\hline CON 04 & 854.69 & 837.50 & 816.41 & 790.63 & 600.00 \\
\hline CON 05 & 668.75 & 655.47 & 639.84 & 621.09 & 734.38 \\
\hline CON 06 & 813.28 & 799.22 & 781.25 & 760.16 & 0 \\
\hline CON 07 & 699.22 & 679.69 & 654.69 & 621.09 & 0 \\
\hline
\end{tabular}

Table 4. PV analysis results of case 3

\begin{tabular}{|c|c|c|c|c|c|}
\hline \multicolumn{7}{|c|}{ Case 3: ZIP = - 0.1:-0.1: 1.2 } \\
\hline CASE & \multicolumn{7}{|c|}{ MAX.INCR.TRANS } \\
\hline TAP & 0.9 & 0.95 & 1.0 & 1.05 & 1.1 \\
\hline BASE CASE & 649.22 & 670.31 & 690.63 & 709.38 & 726.56 \\
\hline CON 01 & 0 & 0 & 0 & 0 & 0 \\
\hline CON 02 & 578.13 & 596.09 & 611.72 & 625.78 & 638.28 \\
\hline CON 03 & 0 & 0 & 0 & 0 & 290.63 \\
\hline CON 04 & 679.69 & 706.25 & 729.69 & 750.78 & 770.31 \\
\hline CON 05 & 550.78 & 567.97 & 583.59 & 598.44 & 611.72 \\
\hline CON 06 & 630.47 & 654.69 & 677.34 & 696.88 & 715.63 \\
\hline CON 07 & 0 & 0 & 0 & 0 & 0 \\
\hline
\end{tabular}


In case 1 , the constant power characteristic is dominant, while constant current and impedance characteristics are higher in case 2. Case 3 does not exist in the actual load. This virtual load characteristic parameter is chosen to analyze the impact of unusual load characteristics, such as when the power demand increases when the voltage is reduced.

Tables 2 to 4 show the PV analysis results for cases 1 to 3, respectively. In cases 1 and 2, the more the tap ratio decreases, the more the maximum incremental transfer power increases. This result is the same as that of the base case with light load. Comparing cases 1 and 2, maximum incremental transfer power of case 2 is always larger than that of case 1 for the same simulation settings. Moreover, in some conditions, such as with tap ratios of 0.95 to 1.05 for contingency 7, the power flow does not converge in case 1, whereas case 2 has the solution. This is because the load is more sensitive to the voltage in case 2 . In other words, the amount of power reduction in case 2 is larger than in case 1 . In case 3 , in contrast to the previous two cases, the maximum incremental transfer power decreased as the tap ratio decreased, as shown in Table 4. This is because that we assumed an unusual load characteristic, such that loads increase when voltage decreases.

The results demonstrate that a reduction in the voltage has a positive impact on the voltage stability of the power system in heavy load and critical contingency conditions.

\section{Conclusion}

PV analysis was performed to analyze the impact of voltage reduction on voltage stability in an emergency case. The simulation procedures and the process to modify the data were explained. The impact of voltage reduction on voltage stability was analyzed in a variety of system conditions and load characteristics. The PV analysis results show that the voltage stability margin was increased when voltage reduction was implemented in all conditions, except for unusual load characteristics. It is concluded that voltage reduction does not cause adverse effects, but instead contributes to improving the voltage stability.

\section{Acknowledgments}

This work was supported by Korea Institute of Energy Technology Evaluation \& Planning (KETEP) grant funded by Korea government Ministry of Trade, Industry \& Energy (No. 20123010020080) and the BK 21 PLUS project.

\section{References}

[1] NERC Standard, Emergency Preparedness and Operations, http://www.nerc.com/pa/Stand/Reliability\%20Standards/Forms/AllItems.aspx.

[2] ERCOT, Operating Guide, Section 4: Emergency Operation, (2010) July, http://www.ercot.com/mktrules/guides/operating/current.html.

[3] PJM, Manual 13 Emergency Operations, Revision 53, (2013) June, http://www.pjm.com/ /media/documents/manuals/m13.ashx.

[4] ISO New England, Operating Procedure 4 - Action During a Capacity Deficiency, (2011) December, http://www.iso-ne.com/rules_proceds/operating/isone/op4/index.html

[5] Alberta ESO, "Emergency System Operations OPP 801 Supply Shortfall", (2012) August, http://www.aeso.ca/downloads/OPP_801.pdf

[6] M. Z. El-Sadek, G. A. Mahmoud, M. M. Dessouky and W. I. Rashed, "Tap changing transformer role in voltage stability enhancement", ELSEVIER. Electric Power Systems Research, vol. 50, (1999), pp. 115.

[7] T. V. Cutsem and R. Mailhot, "Validation of a fast voltage stability analysis method on the Hydro-Qu $\dot{e}$ bec system”, IEEE Transactions on Power Systems, vol. 12, no. 282, (1997).

[8] C. Vournas and M. Karystianos, "Load Tap Changers in Emergency and Preventive Voltage Stability Control", IEEE Transactions on Power Systems, vol. 19, no. 492, (2004). 
[9] S. Greene, I. Dobson and F. L. Alvarado, "Sensitivity of the loading margin to voltage collapse with respect to arbitrary parameters", IEEE Transactions on Power Systems, vol. 12, no. 262, (1997).

[10] S. Arnborg, G. Andersson, D. J. Hill and I. A. Hiskens, "On influence of load modelling for undervoltage load shedding studies", IEEE Transactions on Power Systems, vol. 13, no. 395, (1998).

[11] J. Y. Kim, “An Intelligent System to Prevent Voltage Collapse for A Power System”, Trans. KIEE, vol. 50A, no. 472, (2001).

[12] D. H. Yoo, S. I. Go, C. H. Lee, H. K. Nam, S. J. Ahn and J. H. Choi, "The impact analysis of distribution voltage reduction in voltage stability”, KIEE Summer Conference, Jeju, Korea, (2013) July 10-12 .

[13] L. Lu, "Immediate change in stability and voltage collapse when power system limits are encountered (Master of Sciences)", University of Wisconsin-Madison, Madison, (1991), pp. 2.

[14] P. Kundur, Edited N. J. Balu and M. G. Lauby, "Power System Stability and Control", McGraw-Hill. Inc., New York, (1993).

[15] M. Chakravorty and D. Das, "Voltage stability analysis of radial distribution networks", ELSEVIER, Electrical Power and Energy Systems, vol. 23, no. 130, (2001).

[16] P. Kumkratug, "Power-Voltage Characteristics of Power System with the Short Transmission Line", Am. J. Applied Sciences, vol. 9, no. 907, (2012).

[17] B. Gao, G. K. Morison and P. Kunder, "Towards the development of a systematic approach for voltage stability assessment of large-scale power systems", IEEE Transactions on Power Systems, vol. 11, no. 1316, (1996).

[18] F. A. Shaikh, R. Jain, M. Kotnala and N. Agarwal, "New techniques for the prevention of power system collapse", I. J. Electrical. Electronics Engineering, vol. 1, no. 124, (2012)

[19] P. L. Swe, W. Swe and K. M. Lin, "Effects of tap changing transformer and shunt capacitor on voltage stability enhancement of transmission networks" ,World Academy of Science. Engineering and Technology, vol. 51, (2011), pp. 555-558.

[20] S. P. Moon, B. H. Chang, J. G. Lee and J. S. Choi, "Probabilistic Arrival Power Evaluation considering Voltage Stability", Trans. KIEE, vol. 59, (2010), pp. 1367-1368.

[21] Y. Mansour, Edited L. L. Grigsby, Voltage Stability: The Electric Power Engineering Handbook, CRC Press LLC, Boca Raton, (2001).

[22] Y. H. Kim, H. S. Jung, I. G. Na, S. R. Jo and J. G. Lee, “A study on development of power transfer capability calculation algorithm considering the closest saddle node bifurcation for voltage stability", Trans. KIEE, vol. 52A, no. 558, (2003)

[23] D. H. Yoo, S. J. Ahn and J. H. Choi, "Analysis of the Impact of Voltage Reduction on Voltage Stability using PSS/E”, Workshop on Electrical Engineering 2013, Jeju, Korea, (2013) December 11-13.

[24] S. R. Nam, S. H. Kang, J. H. Lee, E. J. Choi, S. J. Ahn and J. H. Choi, "EMS-Data-Based Load Modeling to Evaluate the Effect of Conservation Voltage Reduction at a National Level", Energies, vol. 6, (2013), pp. 3693-3696.

\section{Authors}

\section{Seon-Ju Ahn}

Seon-Ju Ahn received his B.S., M.S., and Ph.D. degrees in electrical engineering from Seoul National University, Seoul, Korea, in 2002, 2004, and 2009, respectively. $\mathrm{He}$ is an Assistant Professor at Chonnam National University.

\section{Dong-Hyun Yoo}

Dong-Hyun Yoo received his B.S. degrees in electrical engineering from Chonnam National University, Gwang-ju, Korea in 2011. He is currently pursuing the M.S. degree at Chonnam National University.

\section{Joon-Ho Choi}

Joon-Ho Choi received the B.S., M.S. and Ph.D. degrees in Electrical Engineering from Soongsil University, Seoul, Korea in 1996, 1998 and 2002, respectively. He is an Associate Professor at Chonnam National University. 